\title{
Determinantal Representations of the Weighted Core-EP, DMP, MPD, and CMP Inverses
}

\author{
Ivan I. Kyrchei (it) \\ Pidstryhach Institute for Applied Problems of Mechanics and Mathematics, NAS of Ukraine, Lviv, Ukraine \\ Correspondence should be addressed to Ivan I. Kyrchei; st260664@gmail.com
}

Received 31 March 2020; Accepted 2 May 2020; Published 31 May 2020

Academic Editor: George Psihoyios

Copyright (c) 2020 Ivan I. Kyrchei. This is an open access article distributed under the Creative Commons Attribution License, which permits unrestricted use, distribution, and reproduction in any medium, provided the original work is properly cited.

In this paper, new notions of the weighted core-EP left inverse and the weighted MPD inverse which are dual to the weighted coreEP (right) inverse and the weighted DMP inverse, respectively, are introduced and represented. The direct methods of computing the weighted right and left core-EP, DMP, MPD, and CMP inverses by obtaining their determinantal representations are given. A numerical example to illustrate the main result is given.

\section{Introduction}

In the whole article, $\mathbb{R}$ and $\mathbb{C}$ stand for fields of the real and complex numbers, respectively. $\mathbb{C}^{m \times n}$ and $\mathbb{C}_{r}^{m \times n}$ are reserved for the set of all $m \times n$ matrices over $\mathbb{C}$ and its subset of matrices with a rank $r$. For $\mathbf{A} \in \mathbb{C}^{m \times n}$, the symbols $A^{T}, \mathbf{A}^{*}$, and $\operatorname{rk}(\mathbf{A})$ specify the transpose, the conjugate transpose, and the rank of $\mathbf{A}$, respectively. $|\mathbf{A}|$ or $\operatorname{det} \mathbf{A}$ denotes its determinant. A matrix $\mathbf{A} \in \mathbb{C}^{n \times n}$ is Hermitian if $\mathbf{A}^{*}=\mathbf{A}$. The index of $\mathbf{A} \in \mathbb{C}^{n \times n}$, denoted $\operatorname{Ind} \mathbf{A}=k$, is the smallest positive number such that $\operatorname{rk}\left(\mathbf{A}^{k+1}\right)=\operatorname{rk}\left(\mathbf{A}^{k}\right)$.

Definition 1 (see [1]). For $\mathbf{A} \in \mathbb{C}^{m \times n}$ and $\mathbf{W} \in \mathbb{C}^{n \times m}$, the $\mathrm{W}$-weighted Drazin inverse of $\mathbf{A}$ with respect to $\mathbf{W}$, denoted by $\mathbf{A}^{d, W}$, is the unique solution to equations:

$$
\begin{aligned}
(\mathbf{A W})^{k+1} \mathbf{X W} & =(\mathbf{A W})^{k}, \\
\mathbf{X W A W X} & =\mathbf{X}, \\
\mathbf{A W X} & =\mathbf{X W A},
\end{aligned}
$$

where $k=\operatorname{Ind}(\mathbf{A W})$.

The properties of the $\mathrm{W}$-weighted Drazin inverse and some of its applications can be found in [2-8]. Among them, if $\mathbf{A} \in \mathbb{C}^{m \times n}$ with respect to $\mathbf{W} \in \mathbb{C}^{n \times m}$, then

$$
\mathbf{A}_{d, W}=\mathbf{A}\left((\mathbf{W A})^{d}\right)^{2}=\left((\mathbf{A W})^{d}\right)^{2} \mathbf{A} .
$$

Let $\mathbf{A} \in \mathbb{C}^{n \times n}$ and $\mathbf{W}=\mathbf{I}_{n}$ be the identity matrix of order $n$. Then, $\mathbf{X}=\mathbf{A}^{d}$ is the Drazin inverse of $\mathbf{A}$. In particular, if Ind $\mathbf{A}=1$, then the matrix $\mathbf{X}$ is called the group inverse and it is denoted by $\mathbf{X}=\mathbf{A}^{\#}$.

Definition 2 (see [9]). The Moore-Penrose inverse of $\mathbf{A} \in \mathbb{C}^{n \times m}$ is called the exclusive matrix $\mathbf{X}$, denoted by $\mathbf{A}^{\dagger}$, satisfying the following four equations:

$$
\begin{aligned}
\mathbf{A X A} & =\mathbf{A}, \\
\mathbf{X A X} & =\mathbf{X}, \\
(\mathbf{A X})^{*} & =\mathbf{A X}, \\
(\mathbf{X A})^{*} & =\mathbf{X A} .
\end{aligned}
$$

For an arbitrary matrix $\mathbf{A} \in \mathbb{C}^{m \times n}$, it is denoted by

(i) $\mathscr{N}(\mathbf{A})=\left\{\mathbf{x} \in \mathbb{C}^{n \times 1}: \mathbf{A} \mathbf{x}=0\right\}$, the kernel (or the null space) of $\mathbf{A}$

(ii) $\mathscr{C}(\mathbf{A})=\left\{\mathbf{y} \in \mathbb{C}^{m \times 1}: \mathbf{y}=\mathbf{A} \mathbf{x}, \mathbf{x} \in \mathbb{C}^{n \times 1}\right\}$, the column space (or the range space) of $\mathbf{A}$

(iii) $\mathscr{R}(\mathbf{A})=\left\{\mathbf{y} \in \mathbb{C}^{1 \times n}: \mathbf{y}=\mathbf{x A}, \mathbf{x} \in \mathbb{H}^{1 \times m}\right\}$, the row space of $\mathbf{A}$ 
$\mathbf{P}_{A}:=\mathbf{A A}^{\dagger}$ and $\mathbf{Q}_{A}:=\mathbf{A}^{\dagger} \mathbf{A}$ are the orthogonal projectors onto the range of $\mathbf{A}$ and the range of $\mathbf{A}^{*}$, respectively.

For the recent important results regarding generalized inverses see $[10,11]$.

The core inverse was introduced by Baksalary and Trenkler in [12]. Later, it was investigated by Malik and Thome in [13] and Xu et al. in [14], among others.

Definition 3 (see [12]). A matrix $\mathbf{X} \in \mathbb{C}^{n \times n}$ is called the core inverse of $\mathbf{A} \in \mathbb{C}^{n \times n}$ if it satisfies the conditions:

$$
\begin{gathered}
\mathbf{A X}=\mathbf{P}_{A}, \\
\mathscr{C}(\mathbf{X})=\mathscr{C}(\mathbf{A}) .
\end{gathered}
$$

When such matrix $\mathbf{X}$ exists, it is denoted by $\mathbf{A}^{\#}$.

The core inverse was extended to the core-EP inverse defined by Prasad and Mohana [15]. Based on the determinantal representation of an reflexive inverse [16, 17], determinantal formulas for the core-EP inverse have been derived in [15].

Other generalizations of the core inverse were introduced, namely, BT inverses [18], DMP inverses [13], and CMP inverses [19]. The characterizations, computing methods, some applications of the core inverse, and its generalizations were investigated (see, e.g., [20-30]). Recently, determinantal representations of the core inverse and its generalizations have been obtained in both cases for matrices over the field of complex numbers [31] by using usual determinants and over the quaternion skew field [32] by using noncommutative row-column determinants introduced in [33, 34].

Only recent generalizations of the core inverse were extended to rectangular matrices by using the $\mathrm{W}$-weighted Drazin inverse. Among them, the weighted core-EP inverse was introduced in [35], its representations and properties were studied in $[36,37]$, and generalizations of the weighted core-EP inverse were expanded over a ring with involution [38] and Hilbert space [39], respectively. The concepts of the complex weighted DMP and CMP inverses were introduced and explored in [40,41], respectively.

The main goals of this paper are to introduce and represent new notions of the weighted core-EP left inverse and the weighted MPD inverse which are dual to the weighted core-EP (right) inverse and the weighted DMP inverse, respectively. Also, the direct methods of computing the weighted right and left core-EP, DMP, MPD, and CMP inverses by obtaining their determinantal representations are given.

The determinantal representation of the usual inverse is the matrix with cofactors in entries that suggests a direct method of finding the inverse of a matrix. The same is desirable for the generalized inverses. But, there are various expressions of determinantal representations of generalized inverses which are in regard to the search of their more applicable explicit expressions (see, e.g., [16, 17, 42-44]).

In this paper, determinantal representations of a generalized inverse obtained based on their limit representations are used, namely, for the Moore-Penrose inverse in
$[44,45]$, for the Drazin inverse in $[46,47]$, and for the $\mathrm{W}$-weighted Drazin inverse in [48-50].

The paper is organized as follows. Section 2 starts with a preliminary introduction of the determinantal representations of the Moore-Penrose inverse, of the Drazin and weighted Drazin inverses, and of the core inverse and its generalizations. Section 3 introduces the concepts of the left weighted core-EP inverse and gives determinantal representations of both left and right weighted core-EP inverse. In Section 4, the weighted DMP and MPD inverses are established and determinantal representations of the weighted DMP and MPD inverses are obtained. Determinantal representations of the CMP inverse are obtained in Section 5. A numerical example to illustrate the main results is considered in Section 6. Finally, in Section 7, the conclusions are drawn.

\section{Preliminaries}

The following notations for determinantal representations of generalized inverses are used.

Let $\alpha:=\left\{\alpha_{1}, \ldots, \alpha_{k}\right\} \subseteq\{1, \ldots, m\}$ and $\beta:=\left\{\beta_{1}, \ldots\right.$, $\left.\beta_{k}\right\} \subseteq\{1, \ldots, n\}$ be subsets with $1 \leq k \leq \min \{m, n\}$. By $\mathbf{A}_{\beta}^{\alpha}$, denote a submatrix of $\mathbf{A} \in \mathbb{C}^{m \times n}$ with rows and columns indexed by $\alpha$ and $\beta$, respectively. Then, $\mathbf{A}_{\alpha}^{\alpha}$ is a principal submatrix of $\mathbf{A}$ with rows and columns indexed by $\alpha$ and $|\mathbf{A}|_{\alpha}^{\alpha}$ is the corresponding principal minor of the determinant $|\mathbf{A}|$. Suppose that

$$
L_{k, n}:=\left\{\alpha: \alpha=\left(\alpha_{1}, \ldots, \alpha_{k}\right), 1 \leq \alpha_{1}<\cdots<\alpha_{k} \leq n\right\},
$$

stands for the collection of strictly increasing sequences of $1 \leq k \leq n$ integers chosen from $\{1, \ldots, n\}$. For fixed $i \in \alpha$ and $j \in \beta$, put

$$
\begin{aligned}
& I_{r, m}\{i\}:=\left\{\alpha: \alpha \in L_{r, m}, i \in \alpha\right\}, \\
& J_{r, n}\{j\}:=\left\{\beta: \beta \in L_{r, n}, j \in \beta\right\} .
\end{aligned}
$$

$\mathbf{a}_{. j}$ and $\mathbf{a}_{. j}^{*}, \mathbf{a}_{i .}$ and $\mathbf{a}_{i \text {. denote }}^{*}$ the $j$ th columns and the $i$-th rows of $\mathbf{A}$ and $\mathbf{A}^{*}$, respectively. By $\mathbf{A}_{i .}(\mathbf{b})$ and $\mathbf{A}_{j}(\mathbf{c})$, the matrices obtained from $\mathbf{A}$ by replacing its $i$-th row with row $\mathbf{b}$ and its $j$-th column with column $\mathbf{c}$ are denoted.

Lemma 1 (see [44]). If $\mathbf{A} \in \mathbb{C}_{r}^{m \times n}$, then the Moore-Penrose inverse $\mathbf{A}^{\dagger}=\left(\mathbf{a}_{i j}^{\dagger}\right) \in \mathbb{C}^{n \times m}$ possesses the determinantal representations:

$$
\begin{aligned}
a_{i j}^{\dagger} & =\frac{\sum_{\beta \in J_{r, n}\{i\}}\left|\left(\mathbf{A}^{*} \mathbf{A}\right)_{. i}\left(\mathbf{a}_{. j}^{*}\right)\right|_{\beta}^{\beta}}{\sum_{\beta \in J_{r, n}}\left|\mathbf{A}^{*} \mathbf{A}\right|_{\beta}^{\beta}} \\
& =\frac{\sum_{\alpha \in I_{r, m}\{j\}}\left|\left(\mathbf{A A}^{*}\right)_{j .}\left(\mathbf{a}_{i .}^{*}\right)\right|_{\alpha}^{\alpha}}{\sum_{\alpha \in I_{r, m}}\left|\mathbf{A A}^{*}\right|_{\alpha}^{\alpha}} .
\end{aligned}
$$

Remark 1. For an arbitrary full-rank matrix $\mathbf{A} \in \mathbb{C}_{r}^{m \times n}$, a row-vector $\mathbf{b} \in \mathbb{C}^{1 \times m}$, and a column-vector $\mathbf{c} \in \mathbb{C}^{n \times 1}$, it is given as, respectively, 


$$
\begin{aligned}
\left|\left(\mathbf{A A}^{*}\right)_{i .}(\mathbf{b})\right| & =\sum_{\alpha \in I_{m, m},\{i\}}\left|\left(\mathbf{A A}^{*}\right)_{i .}(\mathbf{b})\right|_{\alpha}^{\alpha}, \quad i=1, \ldots, m, \\
\left|\mathbf{A A}^{*}\right| & =\sum_{\alpha \in I_{m, m}}\left|\mathbf{A A}^{*}\right|_{\alpha}^{\alpha}, \quad \text { when } r=m ; \\
\left|\left(\mathbf{A}^{*} \mathbf{A}\right)_{. j}(\mathbf{c})\right| & =\sum_{\beta \in J_{n, n}\{j\}}\left|\left(\mathbf{A}^{*} \mathbf{A}\right)_{. j}(\mathbf{c})\right|_{\beta}^{\beta}, \quad j=1, \ldots, n, \\
\left|\mathbf{A}^{*} \mathbf{A}\right| & =\sum_{\beta \in J_{n, m}}\left|\mathbf{A}^{*} \mathbf{A}\right|_{\beta}^{\beta}, \quad \text { when } r=n .
\end{aligned}
$$

Corollary $\mathbf{1}$ (see [45]). Let $\mathbf{A} \in \mathbb{C}_{r}^{m \times n}$. Then, the following determinantal representations can be obtained:

(i) For the projector $\mathbf{Q}_{A}=\left(q_{i j}\right)_{n \times n}$,

$$
q_{i j}=\frac{\sum_{\beta \in J_{r, n}\{i\}}\left|\left(\mathbf{A}^{*} \mathbf{A}\right)_{. i}\left(\dot{a}_{. j}\right)\right|_{\beta}^{\beta}}{\sum_{\beta \in J_{r, n}}\left|\mathbf{A}^{*} \mathbf{A}\right|_{\beta}^{\beta}}=\frac{\sum_{\alpha \in I_{r, n}\{j\}}\left|\left(\mathbf{A}^{*} \mathbf{A}\right)_{j .}\left(\dot{a}_{i .}\right)\right|_{\alpha}^{\alpha}}{\sum_{\alpha \in I_{r, n}}\left|\mathbf{A}^{*} \mathbf{A}\right|_{\alpha}^{\alpha}},
$$

where $\dot{a}_{. j}$ is the $j$-th column and $\dot{a}_{i .}$ is the $i$-th row of $\mathbf{A}^{*} \mathbf{A}$;

(ii) For the projector $\mathbf{P}_{A}=\left(p_{i j}\right)_{m \times m}$,

$$
p_{i j}=\frac{\sum_{\alpha \in I_{r, m}\{j\}}\left|\left(\mathbf{A A}^{*}\right)_{j} .\left(\ddot{a}_{i .}\right)\right|_{\alpha}^{\alpha}}{\sum_{\alpha \in I_{r, m}}\left|\mathbf{A} \mathbf{A}^{*}\right|_{\alpha}^{\alpha}}=\frac{\sum_{\beta \in J_{r, m}\{i\}}\left|\left(\mathbf{A A}^{*}\right)_{. i}\left(\ddot{a}_{. j}\right)\right|_{\beta}^{\beta}}{\sum_{\beta \in J_{r, m}}\left|\mathbf{A A}^{*}\right|_{\beta}^{\beta}},
$$

where $\ddot{a}_{i .}$ is the $i$-th row and $\ddot{a}_{. j}$ is the $j$-th column of AA*.

Lemma 2 (see [48]). Let $\mathbf{A} \in \mathbb{C}^{m \times n}, \mathbf{W} \in \mathbb{C}^{n \times m}$, and $k=\max \{\operatorname{Ind}(\mathbf{A W})$, Ind (WA) $\}$. Then, $\mathbf{A}_{d, W}=\left(\mathbf{a}_{i j}^{d, W}\right) \in \mathbb{C}^{m \times n}$ can be expressed as

$$
\begin{aligned}
a_{i j}^{d, W} & =\frac{\left.\sum_{\beta \in J_{r, m}\{i\}}(\mid \mathbf{A} \mathbf{W})_{. i}^{k+2}\left(\bar{v}_{. j}\right)\right|_{\beta} ^{\beta}}{\sum_{\beta \in J_{r, m}}\left|(\mathbf{A W})^{k+2}\right|_{\beta}^{\beta}} \\
& =\frac{\sum_{\alpha \in I_{r, n}\{j\}}\left|(\mathbf{W A})_{j .}^{k+2}\left(\bar{u}_{i .}\right)\right|_{\alpha}^{\alpha}}{\sum_{\alpha \in I_{r, n}}\left|(\mathbf{W A})^{k+2}\right|_{\alpha}^{\alpha}}
\end{aligned}
$$

where $\bar{u}_{i .}$ is the $i$-th row of $\overline{\mathbf{U}}=\mathbf{A}(\mathbf{W A})^{k}$ and $\overline{\mathbf{v}}_{. j}$ is the $j$-th column of $\overline{\mathbf{V}}=(\mathbf{A W})^{k} \mathbf{A}$.

The two core inverses are introduced.

Definition 4 (see [12]). A matrix $\mathbf{X} \in \mathbb{C}^{n \times n}$ is said to be the (right) core inverse of $\mathbf{A} \in \mathbb{C}^{n \times n}$ if it satisfies the conditions:

$$
\begin{aligned}
\mathbf{A X} & =\mathbf{P}_{\mathbf{A}}, \\
\mathscr{C}(\mathbf{X}) & =\mathscr{C}(\mathbf{A}) .
\end{aligned}
$$

When such matrix $\mathbf{X}$ exists, it is denoted by $\mathbf{A}$.
Definition 5 (see [31]). A matrix $\mathbf{X} \in \mathbb{C}^{n \times n}$ is said to be the left core inverse of $\mathbf{A} \in \mathbb{C}^{n \times n}$ if it satisfies the conditions:

$$
\begin{gathered}
\mathbf{X A}=\mathbf{Q}_{\mathbf{A}}, \\
\mathscr{R}(\mathbf{X})=\mathscr{R}(\mathbf{A}) .
\end{gathered}
$$

When such matrix $\mathbf{X}$ exists, it is denoted by $\mathbf{A}$.

Similar as in [15], two core-EP inverses are introduced.

Definition 6 (see [15]). A matrix $\mathbf{X} \in \mathbb{C}^{n \times n}$ is said to be the right core-EP inverse of $\mathbf{A} \in \mathbb{C}^{n \times n}$ if it satisfies the conditions:

$$
\begin{aligned}
& \mathbf{X A X}=\mathbf{A}, \\
& \mathscr{C}(\mathbf{X})=\mathscr{C}\left(\mathbf{X}^{*}\right)=\mathscr{C}\left(\mathbf{A}^{d}\right) .
\end{aligned}
$$

It is denoted by $\mathbf{A}^{\dagger}$.

The following lemma gives characterization of the right core-EP inverse.

Lemma 3 (see [15]). Let $\mathbf{A}, \mathbf{X} \in \mathbb{C}^{n \times n}$ be such that Ind $(\mathbf{A})=k$. Then, $\mathbf{X}$ is the right core-EP inverse of $\mathbf{A}$ if and only if $\mathbf{X}$ satisfies the conditions:

$$
\begin{aligned}
\mathbf{X A}^{k+1} & =\mathbf{A}^{k}, \\
\mathbf{A X}^{2} & =X, \\
(\mathbf{A X})^{*} & =\mathbf{A X}, \\
\mathscr{C}(\mathbf{X}) & \subseteq \mathscr{C}\left(\mathbf{A}^{k}\right) .
\end{aligned}
$$

Lemma 4 (see [22], Theorem 2.3). Let $\mathbf{A} \in \mathbb{C}^{n \times n}$ and let $l$ be a nonnegative integer such that $l \geq k=\operatorname{Ind}(\mathbf{A})$. Then, $\mathbf{A}=\mathbf{A}^{d} \mathbf{A}^{l}\left(\mathbf{A}^{l}\right)^{\dagger}$.

Definition 7. A matrix $\mathbf{X} \in \mathbb{C}^{n \times n}$ is said to be the left core-EP inverse of $\mathbf{A} \in \mathbb{C}^{n \times n}$ if it satisfies the conditions:

$$
\begin{aligned}
\mathbf{X A X} & =\mathbf{A}, \\
\mathscr{R}(\mathbf{X}) & =\mathscr{R}\left(\mathbf{X}^{*}\right)=\mathscr{R}\left(\mathbf{A}^{d}\right) .
\end{aligned}
$$

It is denoted by $\mathbf{A}_{\dagger}$.

Remark 2. Since $\mathscr{C}\left(\left(\mathbf{A}^{*}\right)^{d}\right)=\mathscr{R}\left(\mathbf{A}^{d}\right)$, then the left core inverse $\mathbf{A}$ of $\mathbf{A} \in \mathbb{C}^{n \times n}$ is similar to the $*$ core inverse introduced in [15] and the dual core-EP inverse introduced in [30].

Similarly, the following characterization of the left coreEP inverse is obtained.

Lemma 5 (see [30]). Let $\mathbf{X}, \mathbf{A} \in \mathbb{C}^{n \times n}$ and let $l$ be a nonnegative integer such that $l \geq k=\operatorname{Ind}(\mathbf{A})$. The following statements are equivalent:

(i) $\mathbf{X}$ is the left core-EP inverse of $\mathbf{A}$ 
(ii) $\mathbf{A}^{k+1} \mathbf{X}=\mathbf{A}^{k}, \mathbf{X}^{2} \mathbf{A}=\mathbf{X},(\mathbf{X A})^{*}=\mathbf{X A}$, and $\mathscr{R}(\mathbf{X}) \subseteq$ $\mathscr{R}\left(\mathbf{A}^{k}\right)$

(iii) $\mathbf{X}=\mathbf{A}=\left(\mathbf{A}^{l}\right)^{\dagger} \mathbf{A}^{l} \mathbf{A}^{d}$

Thanks to [22], there exists the simple relation between the left and right core-EP inverses, $(\mathbf{A})^{*}=\left(\mathbf{A}^{*}\right)_{\oplus^{*}}$ So, it is enough to investigate the left core-EP inverse, so the right core-EP inverse case can be investigated analogously. But in [31], separate determinantal representations of both core-EP inverses and the both core inverses are given.

Lemma 6 (see [31]). Suppose $\mathbf{A} \in \mathbb{C}^{n \times n}, \quad \operatorname{Ind} \mathbf{A}=k$, $r k\left(\mathbf{A}^{k}\right)=s$, and there exist $\mathbf{A}^{\oplus}=\left(a_{i j}^{\oplus, r}\right)$ and $\mathbf{A}_{\oplus}=\left(a_{i j}^{\oplus, l}\right)$. Then, they have the following determinantal representations, respectively,

$$
\begin{aligned}
& a_{i j}^{\oplus, r}=\frac{\sum_{\alpha \in I_{s, n}\{j\} \mid}\left|\left(\mathbf{A}^{k+1}\left(\mathbf{A}^{k+1}\right)^{*}\right)_{j .}\left(\widehat{a}_{i .}\right)\right|_{\alpha}^{\alpha}}{\sum_{\alpha \in I_{s, n}}\left|\mathbf{A}^{k+1}\left(\mathbf{A}^{k+1}\right)^{*}\right|_{\alpha}^{\alpha}}, \\
& a_{i j}^{\dagger, l}=\frac{\sum_{\beta \in J_{s, n}\{i\}}\left|\left(\left(\mathbf{A}^{k+1}\right)^{*} \mathbf{A}^{k+1}\right)_{. i}\left(\check{\mathbf{a}}_{\cdot j}\right)\right|_{\beta}^{\beta}}{\sum_{\beta \in J_{s, n}}\left|\left(\mathbf{A}^{k+1}\right)^{*} \mathbf{A}^{k+1}\right|_{\beta}^{\beta}},
\end{aligned}
$$

where $\widehat{a}_{i .}$ is the $i$-th row of $\widehat{A}=\mathbf{A}^{k}\left(\mathbf{A}^{k+1}\right)^{*}$ and $\check{\mathbf{a}}_{\text {. }}$ is the $j$-th column of $\check{\mathbf{A}}=\left(\mathbf{A}^{k+1}\right)^{*} \mathbf{A}^{k}$.

If Ind $\mathbf{A}=1$, then $\mathbf{A}^{\boxplus}=\left(\mathbf{a}_{i j}^{\oplus, r}\right)$ and $\mathbf{A}_{\circledast}=\left(\mathbf{a}_{i j}^{\oplus, l}\right)$ have the following determinantal representations, respectively,

$$
\begin{aligned}
& a_{i j}^{\oplus, r}=\frac{\sum_{\alpha \in I_{s, n}\{j\}}\left|\left(\mathbf{A}^{2}\left(\mathbf{A}^{2}\right)^{*}\right)_{j .}\left(\widehat{\mathbf{a}}_{i .}\right)\right|_{\alpha}^{\alpha}}{\sum_{\alpha \in I_{s, n}}\left|\mathbf{A}^{2}\left(\mathbf{A}^{2}\right)^{*}\right|_{\alpha}^{\alpha}}, \\
& a_{i j}^{\oplus, l}=\frac{\sum_{\beta \in J_{s, n}\{i\} \mid}\left|\left(\left(\mathbf{A}^{2}\right)^{*} \mathbf{A}^{2}\right)_{. i}\left(\check{\mathbf{a}}_{. j}\right)\right|_{\beta}^{\beta}}{\sum_{\beta \in J_{s, n}}\left|\left(\mathbf{A}^{2}\right)^{*} \mathbf{A}^{2}\right|_{\beta}^{\beta}},
\end{aligned}
$$

where $\widehat{\mathbf{a}}_{i}$ is the $i$-th row of $\widehat{\mathbf{A}}=\mathbf{A}\left(\mathbf{A}^{2}\right)^{*}$ and $\check{\mathbf{a}}_{. j}$ is the $j$-th column of $\check{\mathbf{A}}=\left(\mathbf{A}^{2}\right)^{*} \mathbf{A}$.

\section{Concepts of the W-Weighted Core-EP Inverses and Their Determinantal Representations}

The concept of the $\mathrm{W}$-weighted core-EP inverse was introduced by Ferreyra et al. in [35].

Definition 8. Suppose $\mathbf{A} \in \mathbb{C}^{m \times n}, \quad \mathbf{W} \in \mathbb{C}^{n \times m}$, and $k=\max \{$ Ind (WA), Ind (AW) $\}$, then the (right) $\mathrm{W}$-weighted core-EP inverse of $\mathbf{A}$ is the unique solution to the system:

$$
\begin{aligned}
& \mathbf{W A W X}=(\mathbf{W A})^{k}\left[(\mathbf{W A})^{k}\right]^{\dagger}, \\
& \mathscr{C}(\mathbf{X}) \subseteq \mathscr{C}\left((\mathbf{A W})^{k}\right) .
\end{aligned}
$$

It is denoted by $\mathbf{A}^{\oplus, W, r}$.

From [35], the right weighted core-EP inverse can be determined as follows.

Lemma 7 (see [35]). Let $\mathbf{A}, \mathbf{X} \in \mathbb{C}^{m \times n}, \mathbf{W} \in \mathbb{C}^{n \times m}$, and $k=\max \{\operatorname{Ind}(\mathbf{W A})$, Ind (AW) $\}$. The following statements are equivalent:

(i) $\mathbf{X}$ is the right weighted core-EP inverse of $\mathbf{A}$.

(ii) $\mathbf{X W} \quad(\mathbf{A W})^{k+1}=(\mathbf{A W})^{k}, \mathbf{A W X W X}=\mathbf{X}$, and (WA $\mathbf{W X})^{*}=\mathbf{W A W X}$.

$$
\mathbf{X}=\mathbf{A}\left[(\mathbf{W A})^{\oplus}\right]^{2}
$$

Introduction of a left weighted core-EP inverse is proposed as well.

Definition 9. Suppose $\mathbf{A} \in \mathbb{C}^{m \times n}, \quad \mathbf{W} \in \mathbb{C}^{n \times m}$, and $k=\max \{$ Ind (WA), Ind (AW) $\}$. The left $\mathbf{W}$-weighted core$\mathrm{EP}$ inverse of $\mathbf{A}$ is the unique solution to the system:

$$
\begin{aligned}
& \mathbf{X W A W}=\left[(\mathbf{A W})^{k}\right]^{\dagger}(\mathbf{A W})^{k}, \\
& \mathscr{R}(\mathbf{X}) \subseteq \mathscr{R}\left((\mathbf{W} \mathbf{A})^{k}\right) .
\end{aligned}
$$

It is denoted by $\mathbf{A}^{\oplus, W, l}$.

Theorem 1. Let $\mathbf{A}, \mathbf{X} \in \mathbb{C}^{m \times n}, \quad \mathbf{W} \in \mathbb{C}^{n \times m}$, and $k=$ $\max \{\operatorname{Ind}(\mathbf{W A})$, Ind $(\mathbf{A W})\}$. The following statements are equivalent:

(i) $\mathbf{X}=\left[(\mathbf{A W})_{\oplus}\right]^{2} \mathbf{A}$.

(ii) $\mathbf{X}$ is the left weighted core-EP inverse of $\mathbf{A}$.

(iii) $\mathbf{X}$ is the unique solution to the three equations:

$$
\begin{aligned}
(\mathbf{W A})^{k+1} \mathbf{W X} & =(\mathbf{W A})^{k}, \\
\mathbf{X W X W A} & =\mathbf{X}, \\
(\mathbf{X W A W})^{*} & =\mathbf{X W A W .}
\end{aligned}
$$

Proof. (i) $\longmapsto$ (ii). It is shown that $\mathbf{X}=\left[(\mathbf{A W})_{\oplus}\right]^{2} \mathbf{A}$ satisfies condition (23). Indeed, 


$$
\begin{aligned}
& \mathbf{X W A W}=\left[(\mathbf{A W})_{\oplus}\right]^{2} \mathbf{A W A W}=(\mathbf{A W})_{\oplus} \mathbf{A W} \\
&=\left[(\mathbf{A W})^{k}\right]^{\dagger}(\mathbf{A W})^{k}(\mathbf{A W})^{d} \mathbf{A W}=\left[(\mathbf{A W})^{k}\right]^{\dagger}(\mathbf{A W})^{k}, \\
& {\left[(\mathbf{A W})_{\oplus}\right]^{2} \mathbf{A} }=\left[(\mathbf{A W})_{\oplus}\right]^{3} \mathbf{A W A}=\cdots=\left[(\mathbf{A W})_{\oplus}\right]^{k+2}(\mathbf{A W})^{k} \mathbf{A} \\
&=\left[(\mathbf{A W})_{\oplus}\right]^{k+2} \mathbf{A}(\mathbf{W} \mathbf{A})^{k}, \quad \text { i.e. } \\
& \mathscr{R}\left(\left[(\mathbf{A W})_{\oplus}\right]^{2} \mathbf{A}\right) \subseteq \mathscr{R}\left((\mathbf{W} \mathbf{A})^{k}\right) .
\end{aligned}
$$

(ii) $\longmapsto$ (iii). Now, it is needed to prove that $\mathbf{X}=\left[(\mathbf{A W})_{\oplus}\right]^{2} \mathbf{A}$ satisfies the equations in (24).

Since $(\mathbf{W A})^{k+1} \mathbf{W}=\mathbf{W}(\mathbf{A W})^{k+1}$ and from Lemma 5,

$$
(\mathbf{A W})_{\oplus}=\left((\mathbf{A W})^{k}\right)^{\dagger}(\mathbf{A W})^{k}(\mathbf{A W})^{d}
$$

then

$$
\begin{aligned}
(\mathbf{W A})^{k+1} \mathbf{W}\left[(\mathbf{A W})_{\oplus}\right]^{2} \mathbf{A} & =\mathbf{W}\left[(\mathbf{A W})^{k+1}(\mathbf{A W})_{\oplus}\right](\mathbf{W A})_{\oplus} \mathbf{A} \\
& =\mathbf{W}(\mathbf{A W})^{k}\left((\mathbf{A W})^{k}\right)^{\dagger}(\mathbf{A W})^{k}(\mathbf{A W})^{d} \mathbf{A} \\
& =\mathbf{W}(\mathbf{A W})^{k}(\mathbf{A W})^{d} \mathbf{A} \\
& =(\mathbf{W A})^{k+1}(\mathbf{W A})^{d} \\
& =(\mathbf{W A})^{k} .
\end{aligned}
$$

By Lemma 5, taking into account $\left[(\mathbf{A W})_{\oplus}\right]^{2}$ $\mathbf{A W}=(\mathbf{A W})_{\oplus}$, it is obtained that

$$
\begin{aligned}
{\left[(\mathbf{A W})_{\oplus}\right]^{2} \mathbf{A W}\left(\left[(\mathbf{A W})_{\oplus}\right]^{2} \mathbf{A W}\right) \mathbf{A} } & =\left[(\mathbf{A W})_{\oplus}\right]^{2} \mathbf{A W}(\mathbf{A W})_{\oplus} \mathbf{A} \\
& =\left[(\mathbf{A W})_{\oplus}\right]^{2} \mathbf{A} .
\end{aligned}
$$

Finally,

$$
\left(\left[(\mathbf{A W})_{\oplus}\right]^{2} \mathbf{A W A W}\right)^{*}=\left((\mathbf{A W})_{\oplus} \mathbf{A W}\right)^{*}=(\mathbf{A W})_{\oplus} \mathbf{A W} .
$$

Now, the uniqueness of $\mathbf{X}$ is proven. Let

$$
\begin{aligned}
(\mathbf{W A})^{k+1} \mathbf{W X} & =(\mathbf{W A})^{k}, \\
\mathbf{X W X W A} & =\mathbf{X} \\
(\mathbf{X W A W})^{*} & =\mathbf{X W A W}, \\
\mathbf{X} & =\left[(\mathbf{A W})_{\oplus}\right]^{2} \mathbf{A} .
\end{aligned}
$$

Suppose there also exists the left weighted core-EP inverse $\mathbf{Y}$ such that

$$
\begin{aligned}
(\mathbf{W A})^{k+1} \mathbf{W Y} & =(\mathbf{W A})^{k}, \\
\mathbf{Y W Y W A} & =\mathbf{Y}, \\
(\mathbf{Y W A W})^{*} & =\text { YWAW } .
\end{aligned}
$$

It is shown that $\mathbf{Y}=\mathbf{X}=\left[(\mathbf{A W})_{\oplus}\right]^{2} \mathbf{A}$. So,

$$
\begin{aligned}
\mathbf{Y} & =\mathbf{Y W Y W A}=\mathbf{Y}(\mathbf{W Y})^{2}(\mathbf{W A})^{2}=\mathbf{Y}(\mathbf{W} \mathbf{Y})^{k}(\mathbf{W A})^{k} \\
& =\mathbf{Y}(\mathbf{W} \mathbf{Y})^{k}(\mathbf{W A})^{k+1} \mathbf{W} \mathbf{X}=(\mathbf{Y W})^{k+1}(\mathbf{A W})^{k+1} \mathbf{X}=(\mathbf{Y W A W})^{k+1} \mathbf{X} \\
& =\left(\left[(\mathbf{A W})^{k}\right]^{\dagger}(\mathbf{A W})^{k}\right)^{k+1} \mathbf{X}=\left[(\mathbf{A W})^{2 k+1}\right]^{\dagger}(\mathbf{A W})^{2 k+1}\left[(\mathbf{A W})_{\oplus}\right]^{2} \mathbf{A} .
\end{aligned}
$$
So,

By Lemma 5, $(\mathbf{A W})_{\oplus}=\left[(\mathbf{A W})^{2 k+1}\right]^{\dagger}(\mathbf{A W})^{2 k+1}(\mathbf{A W})^{d}$.

$$
\begin{aligned}
\mathbf{Y} & =\left[(\mathbf{A W})^{2 k+1}\right]^{\dagger}(\mathbf{A W})^{2 k+1}\left[(\mathbf{A W})^{2 k+1}\right]^{\dagger}(\mathbf{A W})^{2 k+1}(\mathbf{A W})^{d}(\mathbf{A W})_{\oplus} \mathbf{A} \\
& =\left[(\mathbf{A W})^{2 k+1}\right]^{\dagger}(\mathbf{A W})^{2 k+1}(\mathbf{A W})^{d}(\mathbf{A W})_{\oplus} \mathbf{A}=\left[(\mathbf{A W})_{\oplus}\right]^{2} \mathbf{A}
\end{aligned}
$$

Finally, from the uniqueness of $\mathbf{X}$ follows (iii) $\mapsto(\mathrm{i})$.

Now, determinantal representations of the $\mathrm{W}$-weighted core-EP inverses are given.

Theorem 2. Suppose $\mathbf{A} \in \mathbb{C}^{m \times n}, \quad \mathbf{W} \in \mathbb{C}^{n \times m}$, and $k=\max \{\operatorname{Ind}(\mathbf{W A}), \operatorname{Ind}(\mathbf{A W})\}, \quad r k(\mathbf{W A})^{k}=s . \quad$ Denoting $\mathbf{W A}:=\mathbf{U}=\left(u_{i j}\right) \in \mathbb{C}^{n \times n}$. Then, the right weighted core-EP inverse $\mathbf{A}^{\oplus, W, r}=\left(\mathbf{a}_{i j}^{\oplus, W, r}\right) \in \mathbb{C}^{m \times n}$ possesses the determinantal representations:

$$
a_{i j}^{\oplus, W, r}=\frac{\sum_{\alpha \in I_{s, n}\{j\}}\left|\left(\mathbf{U}^{k+1}\left(\mathbf{U}^{k+1}\right)^{*}\right)_{j .}\left(\tilde{\phi}_{i .}\right)\right|_{\alpha}^{\alpha}}{\left(\sum_{\alpha \in I_{s, n}}\left|\mathbf{U}^{k+1}\left(\mathbf{U}^{k+1}\right)^{*}\right|_{\alpha}^{\alpha}\right)^{2}},
$$

where $\widetilde{\phi}_{i}$ is the $i$-th row of $\widetilde{\Phi}=\Phi \mathbf{U}^{k}\left(\mathbf{U}^{k+1}\right)^{*}$. The matrix $\Phi=$ $\left(\phi_{i f}\right)$ is determined as follows:

$$
\phi_{i f}=\sum_{\alpha \in I_{s, n}\{f\}}\left|\left(\mathbf{U}^{k+1}\left(\mathbf{U}^{k+1}\right)^{*}\right)_{f .}\left(\widetilde{u}_{i .}\right)\right|_{\alpha}^{\alpha},
$$

where $\widetilde{u}_{i}$ is the $i$-th row of $\widetilde{U}=\mathbf{A} \mathbf{U}^{k}\left(\mathbf{U}^{k+1}\right)^{*}$.

Proof. Taking into account (22) for $\mathbf{A}^{\oplus, W, r}$, it is obtained that

$$
a_{i j}^{\oplus, W, r}=\sum_{l=1}^{n} \sum_{f=1}^{n} \mathbf{a}_{i l} \mathbf{u}_{l f}^{\oplus, r} \mathbf{u}_{f j}^{\oplus, r}
$$

Using the determinantal representation (18) of $\mathbf{U}^{\oplus}$ gives 


$$
\begin{aligned}
\phi_{i f} & =\sum_{l=1}^{n} \mathbf{a}_{i l} \mathbf{u}_{l f}^{\oplus, r}=\frac{\sum_{l=1}^{n} \mathbf{a}_{i l} \sum_{\alpha \in I_{s, n}}\{f\}\left|\left(\mathbf{U}^{k+1}\left(\mathbf{U}^{k+1}\right)^{*}\right)_{f .}\left(\widehat{\mathbf{u}}_{l .}\right)\right|_{\alpha}^{\alpha}}{\sum_{\alpha \in I_{s, n} \mid}\left|\mathbf{U}^{k+1}\left(\mathbf{U}^{k+1}\right)^{*}\right|_{\alpha}^{\alpha}} \\
& =\frac{\sum_{\alpha \in I_{s, n}\{f\}}\left|\left(\mathbf{U}^{k+1}\left(\mathbf{U}^{k+1}\right)^{*}\right)_{f .}\left(\widetilde{\mathbf{u}}_{i .}\right)\right|_{\alpha}^{\alpha}}{\sum_{\alpha \in I_{s, n} \mid}\left|\mathbf{U}^{k+1}\left(\mathbf{U}^{k+1}\right)^{*}\right|_{\alpha}^{\alpha}}
\end{aligned}
$$

where $\widehat{\mathbf{u}}_{l .}$ is the $l$-th row of $\widehat{\mathbf{U}}=\mathbf{U}^{k}\left(\mathbf{U}^{k+1}\right)^{*}$ and $\widetilde{u}_{i}$ is the $i$-th row of $\tilde{U}=\mathbf{A U}^{k}\left(\mathbf{U}^{k+1}\right)^{*}$. Denoting

$$
\phi_{i f}=\sum_{\alpha \in I_{s, n}\{f\}}\left|\left(\mathbf{U}^{k+1}\left(\mathbf{U}^{k+1}\right)^{*}\right)_{f .}\left(\widetilde{u}_{i .}\right)\right|_{\alpha}^{\alpha} .
$$

Substituting $\phi_{i f}$ into (36) gives

$$
a_{i j}^{\oplus, W, r}=\sum_{l=1}^{n} \phi_{i f} u_{f j}^{\oplus r r}=\frac{\sum_{f=1}^{n} \phi_{i f} \sum_{\alpha \in I_{s, n}\{f\}}\left|\left(\mathbf{U}^{k+1}\left(\mathbf{U}^{k+1}\right)^{*}\right)_{j .}\left(\widehat{\mathbf{u}}_{f .}\right)\right|_{\alpha}^{\alpha}}{\left(\sum_{\alpha \in I_{s, n}}\left|\mathbf{U}^{k+1}\left(\mathbf{U}^{k+1}\right)^{*}\right|_{\alpha}^{\alpha}\right)^{2}} .
$$

Putting $\sum_{f=1}^{n} \phi_{i f} \widehat{u}_{f}=\tilde{\phi}_{i}$ as the $i$-th row of $\widetilde{\Phi}=\Phi \mathbf{U}^{k}\left(\mathbf{U}^{k+1}\right)^{*}$ yields (34).

Similarly, theorem on the determinantal representation of the left $\mathrm{W}$-weighted core-EP inverse can be proved.

Theorem 3. Suppose $\mathbf{A} \in \mathbb{C}^{m \times n}, \quad \mathbf{W} \in \mathbb{C}^{n \times m}$, and $k=\max \{\operatorname{Ind}(\mathbf{W A}) \operatorname{Ind}(\mathbf{A W})\}, \quad r k(\mathbf{A W})^{k}=s . \quad$ Denoting AW: $=\mathbf{V}=\left(v_{i j}\right) \in \mathbb{C}^{m \times m}$. Then, the left $W$-weighted core$E P$ inverse $\mathbf{A}^{\dagger, W, l}=\left(a_{i j}^{\dagger, W, l}\right) \in \mathbb{C}^{n \times m}$ possesses the determinantal representations

$$
a_{i j}^{\dagger, W, l}=\frac{\sum_{\left.\beta \in J_{s, m} i\right\}}\left|\left(\left(\mathbf{V}^{k+1}\right)^{*} \mathbf{V}^{k+1}\right)_{. i}\left(\widetilde{\psi}_{. j}\right)\right|_{\beta}^{\beta}}{\left(\sum_{\beta \in J_{s, m}}\left|\left(\mathbf{V}^{k+1}\right)^{*} \mathbf{V}^{k+1}\right|_{\beta}^{\beta}\right)^{2}}
$$

where $\widetilde{\psi}_{. j}$ is the $j$-th column of $\widetilde{\Psi}=\left(\mathbf{V}^{k+1}\right)^{*} \mathbf{V}^{k} \Psi$. The matrix is determined, $\Psi=\left(\psi_{l j}\right) \in \mathbb{C}^{m \times n}$, as follows:

$$
\psi_{l j}=\sum_{\beta \in J_{s, m}\{l\}}\left|\left(\left(\mathbf{V}^{k+1}\right)^{*} \mathbf{V}^{k+1}\right)_{. l}\left(\widetilde{v}_{. j}\right)\right|_{\beta}^{\beta},
$$

where $\widetilde{v}_{. j}$ is the $j$-th column of $\tilde{V}=\left(\mathbf{V}^{k+1}\right)^{*} \mathbf{V}^{k} \mathbf{A}$.

\section{The W-Weighted DMP and MPD Inverses and Their Determinantal Representations}

The concept of the DMP inverse was introduced by Malik and Thome as follows.

Definition 10 (see [13]). Suppose that $\mathbf{A} \in \mathbb{C}^{n \times n}$ and Ind $\mathbf{A}=k$. A matrix $\mathbf{X} \in \mathbb{C}^{n \times n}$ is said to be the DMP inverse of $\mathbf{A}$ if it satisfies the conditions:

$$
\begin{aligned}
\mathbf{X A X} & =\mathbf{X}, \\
\mathbf{X A} & =\mathbf{A}^{d} \mathbf{A}, \\
\mathbf{A}^{k} \mathbf{X} & =\mathbf{A}^{k} \mathbf{A}^{\dagger} .
\end{aligned}
$$

It is denoted by $\mathbf{A}^{d, \dagger}$.

It is proved in [13] that if a matrix satisfies the system of equations (42), then it is unique and has the representation $\mathbf{A}^{d, \dagger}=\mathbf{A}^{d} \mathbf{A A}^{\dagger}$.

In [31], the MPD inverse is introduced.

Definition 11. Suppose $\mathbf{A} \in \mathbb{C}^{n \times n}$ and Ind $\mathbf{A}=k$. A matrix $\mathbf{X} \in \mathbb{C}^{n \times n}$ is said to be the MPD inverse of $\mathbf{A}$ if it satisfies the conditions:

$$
\begin{aligned}
\mathbf{X A X} & =\mathbf{X}, \\
\mathbf{A X} & =\mathbf{A A}^{d}, \\
\mathbf{X A}^{k} & =\mathbf{A}^{\dagger} \mathbf{A}^{k} .
\end{aligned}
$$

It is denoted by $\mathbf{A}^{\dagger, d}$.

It is not difficult to show that $\mathbf{A}^{\dagger, d}$ is unique and it can be represented as $\mathbf{A}^{\dagger, d}=\mathbf{A}^{\dagger} \mathbf{A} \mathbf{A}^{d}$.

In [31], the determinantal representations of the DMP and MPD inverses are given.

Recently in [40], the definition of the DMP inverse of a square matrix was extended to rectangular matrices.

Definition 12 (see [40]). Let $\mathbf{A} \in \mathbb{C}^{m \times n}$ and $\mathbf{W} \in \mathbb{C}^{n \times m}$ be a nonzero matrix. The $\mathrm{W}$-weighted DMP (WDMP) inverse of A with respect to $\mathbf{W}$ is defined as

$$
\mathbf{A}^{d, \dagger, W}=\mathbf{W A}^{d, W} \mathbf{W A A}^{\dagger} \text {. }
$$

Lemma 8 (see [40]). Let $\mathbf{A} \in \mathbb{C}^{m \times n}, \mathbf{W} \in \mathbb{C}^{n \times m}$ be a nonzero matrix, and $k=\max \{\operatorname{Ind}(\mathbf{W A})$ Ind $(\mathbf{A W})\}$. The matrix $\mathbf{X}=$ $\mathbf{A}^{d, t, W}$ is the unique matrix that satisfies the following system of equations:

$$
\begin{aligned}
\mathbf{X A X} & =\mathbf{X}, \\
\mathbf{X A} & =\mathbf{W A}^{d, W} \mathbf{W A}, \\
(\mathbf{W A})^{k+1} \mathbf{X} & =(\mathbf{W A})^{k+1} \mathbf{A}^{\dagger} .
\end{aligned}
$$
as well.

Introduction of the weighted MPD inverse is proposed

Theorem 4. Let $\mathbf{A} \in \mathbb{C}^{m \times n}, \mathbf{W} \in \mathbb{C}^{n \times m}$ be a nonzero matrix, and $k=\max \{\operatorname{Ind}(\mathbf{W A})$, Ind $(\mathbf{A W})\}$. Then, the matrix $\mathbf{X}=$ $\mathbf{A}^{\dagger} \mathbf{A W} \mathbf{A}^{d, W} \mathbf{W}$ is the unique solution to the equations:

$$
\begin{aligned}
\mathbf{X A X} & =\mathbf{X}, \\
\mathbf{A X} & =\mathbf{A W A}^{d, W} \mathbf{W}, \\
\mathbf{X}(\mathbf{A W})^{k+1} & =\mathbf{A}^{\dagger}(\mathbf{A W})^{k+1} .
\end{aligned}
$$


Proof. From Definitions 1 and 2 and taking into account (2),

it follows

$$
\begin{aligned}
\mathbf{A}^{\dagger} \mathbf{A} \mathbf{W} \mathbf{A}^{d, W} \mathbf{W}\left(\mathbf{A A}^{\dagger} \mathbf{A}\right) \mathbf{W} \mathbf{A}^{d, W} \mathbf{W} & =\mathbf{A}^{\dagger} \mathbf{A} \mathbf{W}\left(\mathbf{A}^{d, W} \mathbf{W A W A}^{d, W}\right) \mathbf{W} \\
& =\mathbf{A}^{\dagger} \mathbf{A} \mathbf{W} \mathbf{A}^{d, W} \mathbf{W}, \\
\mathbf{A A}^{\dagger} \mathbf{A} \mathbf{W} \mathbf{A}^{d, W} \mathbf{W} & =\mathbf{A} \mathbf{W} \mathbf{A}^{d, W} \mathbf{W}, \\
\mathbf{A}^{\dagger} \mathbf{A} \mathbf{W} \mathbf{A}^{d, W} \mathbf{W}(\mathbf{A W})^{k+1} & =\mathbf{A}^{\dagger} \mathbf{A W}\left((\mathbf{A W})^{d}\right)^{2} \mathbf{A W}(\mathbf{A W})^{k+1} \\
& =\mathbf{A}^{\dagger} \mathbf{A W}(\mathbf{A W})^{d} \mathbf{A W}(\mathbf{A W})^{d}(\mathbf{A W})^{k+1} \\
& =\mathbf{A}^{\dagger}(\mathbf{A W})^{k+1} .
\end{aligned}
$$

It means that $\mathbf{X}=\mathbf{A}^{\dagger} \mathbf{A} \mathbf{W} \mathbf{A}^{d, W} \mathbf{W}$ is the solution to equation (46).

To prove uniqueness, suppose both $\mathbf{X}_{1}$ and $\mathbf{X}_{2}$ are two solutions to (46). Using repeated applications of the equations in (46) and in Definition 1, it is obtained that

$$
\begin{aligned}
\mathbf{X}_{1} & =\mathbf{X}_{1} \mathbf{A} \mathbf{X}_{1}=\mathbf{X}_{1} \mathbf{A} \mathbf{W} \mathbf{A}^{d, W} \mathbf{W}=\mathbf{X}_{1}(\mathbf{A W})^{2}\left(\mathbf{A}^{d, W} \mathbf{W}\right)^{2} \\
& =\cdots=\mathbf{X}_{1}(\mathbf{A W})^{k+1}\left(\mathbf{A}^{d, W} \mathbf{W}\right)^{k+1}=\mathbf{A}^{\dagger}(\mathbf{A W})^{k+1}\left(\mathbf{A}^{d, W} \mathbf{W}\right)^{k+1} \\
& =\mathbf{X}_{2}(\mathbf{A W})^{k+1}\left(\mathbf{A}^{d, W} \mathbf{W}\right)^{k+1}=\mathbf{X}_{2} \mathbf{A} \mathbf{W} \mathbf{A}^{d, W} \mathbf{W}=\mathbf{X}_{2} \mathbf{A} \mathbf{X}_{2}=\mathbf{X}_{2} .
\end{aligned}
$$

It completes the proof.

Definition 13. Let $\mathbf{A} \in \mathbb{C}^{m \times n}$ and $\mathbf{W} \in \mathbb{C}^{n \times m}$ be a nonzero matrix. The $\mathrm{W}$-weighted MPD (WMPD) inverse of $\mathbf{A}$ with respect to $\mathbf{W}$ is defined as

$$
\mathbf{A}^{\dagger, d, W}=\mathbf{A}^{\dagger} \mathbf{A} \mathbf{W} \mathbf{A}^{d, W} \mathbf{W} .
$$

Now, determinantal representations of the WDMP inverse are given.

Theorem 5. Let $\mathbf{A} \in \mathbb{C}_{r}^{m \times n}$ and $\mathbf{W} \in \mathbb{C}^{n \times m}$ be a nonzero matrix. Suppose $k=\max \{\operatorname{Ind}(\mathbf{W A}), \operatorname{Ind}(\mathbf{A W})\}$, and $r k(\mathbf{W A})^{k}=r k \mathbf{U}^{k}=r_{1}$. Then, the determinantal representations of its WDMP inverse $\mathbf{A}^{d, \dagger, W}=\left(a_{i j}^{d, \dagger, W}\right)$ can be expressed as

$$
a_{i j}^{d, \dagger, W}=\frac{\sum_{\alpha \in I_{r, m}\{j\}}\left|\left(\mathbf{A A}^{*}\right)_{j .}\left(\widetilde{\omega}_{i .}\right)\right|_{\alpha}^{\alpha}}{\sum_{\alpha \in I_{r, m}}\left|\mathbf{A} \mathbf{A}^{*}\right|_{\alpha}^{\alpha}\left|(\mathbf{W A})^{k+2}\right|_{\alpha}^{\alpha}}
$$

where $\widetilde{\omega}_{i .}$ is the $i$-th row of $\widetilde{\Omega}=\Omega \mathbf{W A A}^{*}$. The matrix $\Omega=$ $\left(\omega_{i s}\right)$ is such that

$$
\omega_{i s}:=\sum_{\alpha \in I_{r_{1}, n}\{s\}}\left|(\mathbf{W A})_{s .}^{k+2}\left(\widehat{u}_{i .}\right)\right|_{\alpha}^{\alpha},
$$

where $\widehat{\mathbf{u}}_{i}$ is the $i$-th row of $\widehat{\mathbf{U}}=(\mathbf{W A})^{k+1}$.

Proof. Taking into account (42), the following is obtained:

$$
a_{i j}^{d, \dagger, W}=\sum_{t=1}^{m} \sum_{s=1}^{n} \sum_{f=1}^{m} w_{i t} a_{t s}^{d, W} w_{s f} p_{f j}^{A},
$$

where $\mathbf{A}^{d, W}=\left(a_{t s}^{d, W}\right) \in \mathbb{C}^{m \times n}$ and $\mathbf{P}_{A}=\left(p_{f j}^{A}\right) \in \mathbb{C}^{m \times m}$. Applying the determinantal representations (12) of $\mathbf{A}^{d, W}$ and (10) of $\mathbf{P}_{A}$ in (52) gives

$$
a_{i j}^{d, \dagger, W}=\frac{\sum_{s=1}^{n} \sum_{\alpha \in I_{r_{1}, n}\{s\}}\left|(\mathbf{W A})_{s .}^{k+2}\left(\widehat{\mathbf{u}}_{i .}\right)\right|_{\alpha}^{\alpha} \sum_{\alpha \in I_{r, m}}\{j\}\left|\left(\mathbf{A A}^{*}\right)_{j .}\left(\mathbf{w}_{s .}^{(2)}\right)\right|_{\alpha}^{\alpha}}{\left.\sum_{\alpha \in I_{r_{1}, n} \mid} \mid \mathbf{W A}\right)\left.^{k+2}\right|_{\alpha} ^{\alpha} \sum_{\alpha \in I_{r, m}}\left|\mathbf{A A}^{*}\right|_{\alpha}^{\alpha}},
$$

where $\widehat{\mathbf{u}}_{i}$ is the $i$-th row of $\widehat{\mathbf{U}}:=(\mathbf{W A})^{k+1} \in \mathbb{M}^{n \times n}$ and $\mathbf{w}_{s .}^{(2)}$ is the $s$-th row of $\mathbf{W}_{2}=\mathbf{W A A}^{*}$.

Denote

$$
\omega_{i s}:=\sum_{\alpha \in I_{r_{1}, n}\{s\}}\left|(\mathbf{W A})_{s .}^{k+2}\left(\widehat{\mathbf{u}}_{i .}\right)\right|_{\alpha}^{\alpha},
$$

and construct the matrix $\Omega=\left(\omega_{i s}\right)$. Since

$$
\sum_{s=1}^{n} \omega_{i s} \sum_{\alpha \in I_{r, m}\{j\}}\left|\left(\mathbf{A A}^{*}\right)_{j .}\left(w_{s .}^{(2)}\right)\right|_{\alpha}^{\alpha}=\sum_{\alpha \in I_{r, m}\{j\}}\left|\left(\mathbf{A A}^{*}\right)_{j .}\left(\widetilde{\omega}_{i .}\right)\right|_{\alpha}^{\alpha},
$$

where $\widetilde{\omega}_{i .}$ is the $i$-th row of $\widetilde{\Omega}=\Omega \mathbf{W}_{2}=\Omega(\mathbf{W A})^{k+1} \mathbf{A}^{*}$, then, finally, from (53), it follows (50).

It is obtained similarly for the weighted MPD inverse.

Theorem 6. Let $\mathbf{A} \in \mathbb{C}_{r}^{m \times n}$ and $\mathbf{W} \in \mathbb{C}^{n \times m}$ be a nonzero matrix. Suppose $k=\max \{\operatorname{Ind}(\mathbf{W A}), \operatorname{Ind}(\mathbf{A W})\}$, and $r k(\mathbf{A W})^{k}=r k \mathbf{V}^{k}=r_{1}$. Then, the determinantal representations of its WMPD inverse $\mathbf{A}^{\dagger, d, W}=\left(a_{i j}^{\dagger, d, W}\right)$ can be expressed as follows:

$$
a_{i j}^{\dagger, d, W}=\frac{\sum_{\beta \in J_{r, n}\{i\}}\left|\left(\mathbf{A}^{*} \mathbf{A}\right)_{. i}\left(\widetilde{v}_{. j}\right)\right|_{\beta}^{\beta}}{\sum_{\beta \in J_{r, n}}\left|\mathbf{A}^{*} \mathbf{A}\right|_{\beta}^{\beta}\left(\sum_{\beta \in J_{r_{1}, m}}\left|(\mathbf{A W})^{k+2}\right|_{\beta}^{\beta}\right)^{2}},
$$

where $\widetilde{v}_{. j}$ is the $j$-th column of $\widetilde{\Upsilon}=\mathbf{A}^{*} \mathbf{A W} \Upsilon$. The matrix $\Upsilon=$ $\left(v_{s j}\right)$ is determined by 


$$
v_{s j}=\sum_{\beta \in J_{r_{1}, m}\{s\}}\left|(\mathbf{A W})_{. s}^{k+2}\left(\widetilde{\mathbf{v}}_{. j}\right)\right|_{\beta}^{\beta},
$$

where $\widetilde{\mathbf{v}}_{. j}$ is the $j$-th column of $\widetilde{\mathbf{V}}=(\mathbf{A W})^{k+1}$.

Proof. Taking into account (45), the following is obtained:

$$
a_{i j}^{\dagger, d, W}=\sum_{t=1}^{m} \sum_{s=1}^{n} \sum_{f=1}^{m} q_{i f}^{A} w_{f t} a_{t s}^{d, W} w_{s f}
$$

where $\mathbf{A}^{d, W}=\left(a_{t s}^{d, W}\right) \in \mathbb{C}^{m \times n}$ and $\mathbf{Q}_{A}=\left(q_{i f}^{A}\right) \in \mathbb{C}^{n \times n}$. Applying the determinantal representations (11) of $\mathbf{A}^{d, W}$ and (9) of $\mathbf{Q}_{A}$ in (58) gives

$$
a_{i j}^{\dagger, d, W}=\sum_{t=1}^{m} \frac{\sum_{\beta \in J_{r, n}\{i\}}\left|\left(\mathbf{A}^{*} \mathbf{A}\right)_{. i}\left(w_{. t}^{(2)}\right)\right|_{\beta}^{\beta}}{\sum_{\beta \in J_{r, n}}\left|\mathbf{A}^{*} \mathbf{A}\right|_{\beta}^{\beta}} \frac{\sum_{\beta \in J_{r_{1}, m}\{t\}}\left|(\mathbf{A W})_{. t}^{k+2}\left(\widetilde{\mathbf{v}}_{. j}\right)\right|_{\beta}^{\beta}}{\sum_{\beta \in J_{r_{1}, m}}\left|(\mathbf{A W})^{k+2}\right|_{\beta}^{\beta}},
$$

where $w_{. t}^{(2)}$ is the $t$-th column of $\mathbf{W}_{2}=\mathbf{A}^{*} \mathbf{A} \mathbf{W} \in \mathbb{C}^{m \times m}$ and $\widetilde{\mathbf{v}}_{\text {.j }}$ is the $j$-th column of $\widetilde{\mathbf{V}}=(\mathbf{A W})^{k+1}$. Now, construct the matrix $\Upsilon=\left(v_{t j}\right)$, where

$$
v_{t j}:=\sum_{\beta \in J_{r_{1}, m}\{t\}}\left|(\mathbf{A W})_{. t}^{k+2}\left(\widetilde{\mathbf{v}}_{. j}\right)\right|_{\beta}^{\beta} .
$$

Then, from (59) it follows (56).

Theorems 5 and 6 give the determinantal representations of the weighted DMP and MPD inverses. For better understanding, the algorithm of finding one of them, for example, the WDMP inverse from Theorem 5, is presented.

\section{Algorithm 1.}

(1) Compute the matrix $\check{\mathbf{U}}=\mathbf{U}^{k+1}=(\mathbf{W A})^{k+1}$.

(2) By (51), find $\omega_{i s}$ for all $i, s=1, \ldots, n$ and construct the matrix $\Omega=\left(\omega_{i s}\right)$.

(3) Compute the matrix $\widetilde{\Omega}:=\Omega \mathbf{W A A} \mathbf{A}^{*}$.

(4) Finally, find $a_{i j}^{d, \dagger, W}$ by (50) for all $i=1, \ldots, m$ and $j=1, \ldots, n$.

\section{Determinantal Representations of the Weighted CMP Inverse}

In [19], Mehdipour and Salemi investigated the CMP inverse.

Definition 14 (see [19]). Suppose $\mathbf{A} \in \mathbb{C}^{n \times n}$ has the corenilpotent decomposition $\mathbf{A}=\mathbf{A}_{1}+\mathbf{A}_{2}$, where Ind $\mathbf{A}_{1}=$ Ind $\mathbf{A}, \mathbf{A}_{2}$ is nilpotent, and $\mathbf{A}_{1} \mathbf{A}_{2}=\mathbf{A}_{2} \mathbf{A}_{1}=0$. The CMP inverse of $\mathbf{A}$ is called the matrix $\mathbf{A}^{c, \dagger}$ : $=\mathbf{A}^{\dagger} \mathbf{A}_{1} \mathbf{A}^{\dagger}$.

Lemma 9 (see [19]). Let $\mathbf{A} \in \mathbb{C}^{n \times n}$. The matrix $\mathbf{X}=\mathbf{A}^{c, t}$ is the unique matrix that satisfies the following system of equations:

$$
\begin{aligned}
\mathbf{X A X} & =\mathbf{X}, \\
\mathbf{A X A} & =\mathbf{A}_{1}, \\
\mathbf{A X} & =\mathbf{A}_{1} \mathbf{A}^{\dagger}, \\
\mathbf{X A} & =\mathbf{A}^{\dagger} \mathbf{A}_{1} .
\end{aligned}
$$

Moreover, $\mathbf{A}^{c, \dagger}=\mathbf{A}^{\dagger} \mathbf{A} \mathbf{A}^{d} \mathbf{A} \mathbf{A}^{\dagger}$.

Determinantal representations of the CMP inverse are derived in [31].

Recently, Mosić [41] introduced the weighted CMP inverse of a rectangular matrix.

Lemma 10 (see [41]). Let $\mathbf{A} \in \mathbb{C}^{m \times n}$ and $\mathbf{W} \in \mathbb{C}^{n \times m}$ be a nonzero matrix. The system of equations

$$
\begin{aligned}
\mathbf{X A X} & =\mathbf{X}, \\
\mathbf{A X} & =\mathbf{A W A}^{d, W} \mathbf{W A A}^{\dagger}, \\
\mathbf{X A} & =\mathbf{A}^{\dagger} \mathbf{A} \mathbf{W} \mathbf{A}^{d, W} \mathbf{W A},
\end{aligned}
$$

is consistent, and its unique solution is $\mathbf{X}=\mathbf{A}^{\dagger} \mathbf{A} \mathbf{W} \mathbf{A}^{d, W} \mathbf{W A A}^{\dagger}$.

Definition 15. Let $\mathbf{A} \in \mathbb{C}^{m \times n}$ and $\mathbf{W} \in \mathbb{C}^{n \times m}$ be a nonzero matrix. The weighted CMP (WCMP) inverse of $\mathbf{A}$ with respect to $\mathbf{W}$ is defined as

$$
\mathbf{A}^{c, \dagger, W}=\mathbf{A}^{\dagger} \mathbf{A} \mathbf{W} \mathbf{A}^{d, W} \mathbf{W} \mathbf{A} \mathbf{A}^{\dagger} \text {. }
$$

Theorem 7. Let $\mathbf{A} \in \mathbb{C}_{r}^{m \times n}$ and $\mathbf{W} \in \mathbb{C}^{n \times m}$ be a nonzero matrix. Suppose $k=\max \{\operatorname{Ind}(\mathbf{W A}), \operatorname{Ind}(\mathbf{A W})\}$. Then, the determinantal representations of its WCMP inverse $\mathbf{A}^{c, t, W}=$ $\left(a_{i j}^{c, t, W}\right)$ can be expressed as follows:

(i) If $r k(\mathbf{W A})^{k}=r_{1}$, then

$$
a_{i j}^{c, \dagger, W}=\frac{\sum_{\alpha \in I_{r, m}\{j\}}\left|\left(\mathbf{A A}^{*}\right)_{j .}\left(\widetilde{\omega}_{i .}\right)\right|_{\alpha}^{\alpha}}{\left(\sum_{\beta \in J_{r, n}}\left|\mathbf{A}^{*} \mathbf{A}\right|_{\beta}^{\beta}\right)^{2} \sum_{\alpha \in I_{r_{1}, n}}\left|(\mathbf{W} \mathbf{A})^{k+2}\right|_{\alpha}^{\alpha}},
$$

where $\widetilde{\omega}_{i}$ is the $i$-th row of $\widetilde{\Omega}=\Omega \mathbf{W A A} *$. The matrix $\Omega=\left(\omega_{i s}\right)$ is such that

$$
\omega_{i s}=\sum_{\alpha \in I_{r_{1}, n}\{s\}}\left|(\mathbf{W A})_{s .}^{k+2}\left(\phi_{i .}^{(1)}\right)\right|_{\alpha}^{\alpha} .
$$

Here, $\phi_{i}^{(1)}$ is the $i$-th row of $\Phi_{1}=\Phi \mathbf{A}(\mathbf{W A})^{k}$ and the matrix $\Phi=\left(\phi_{i t}\right)$ is such that

$$
\phi_{i t}:=\sum_{\beta \in J_{r, n}\{i\}}\left|\left(\mathbf{A}^{*} \mathbf{A}\right)_{. i}\left(\mathbf{w}_{. t}^{(1)}\right)\right|_{\beta}^{\beta},
$$

where $\mathbf{w}_{. t}^{(1)}$ is the t-th column of $\mathbf{W}_{1}=\mathbf{A}^{*} \mathbf{A W}$.

(ii) If $r k(\mathbf{A W})^{k}=r_{1}$, then 


$$
a_{i j}^{c, \dagger, W}=\frac{\sum_{\beta \in J_{r, n}\{i\}}\left|\left(\mathbf{A}^{*} \mathbf{A}\right)_{. i}\left(\widetilde{v}_{. j}\right)\right|_{\beta}^{\beta}}{\left(\sum_{\alpha \in I_{r, m}}\left|\mathbf{A A}^{*}\right|_{\alpha}^{\alpha}\right)^{2} \sum_{\beta \in J_{r_{1}, m}}\left|(\mathbf{A W})^{k+2}\right|_{\beta}^{\beta}},
$$

where $\widetilde{v}_{. j}$ is the $j$-th column of $\widetilde{Y}=\mathbf{A}^{*} \mathbf{A W} \Upsilon$. The matrix $\Upsilon=\left(v_{t j}\right)$ is determined by

$$
v_{t j}=\sum_{\beta \in J_{r_{1}, m}\{t\}}\left|(\mathbf{A W})_{. t}^{k+2}\left(\psi_{. j}^{(1)}\right)\right|_{\beta}^{\beta},
$$

where $\psi_{. j}^{(1)}$ is the $j$-th column of $\Psi^{(1)}=(\mathbf{A W})^{k} \mathbf{A} \Psi$. Here, $\Psi=\left(\psi_{s j}\right)$ is such that

$$
\psi_{s j}:=\sum_{\alpha \in I_{r, m}\{j\}}\left|\left(\mathbf{A A}^{*}\right)_{j .}\left(\mathbf{w}_{s .}^{(2)}\right)\right|_{\alpha}^{\alpha},
$$

where $\mathbf{w}_{s .}^{(2)}$ is the $s$-th row of $\mathbf{W}_{2}=\mathbf{W A A}^{*}$.

Proof

(i) Taking into account (63) and applying one of the cases of (9) and (10) for the determinantal representations of $\mathbf{Q}_{A}$ and $\mathbf{P}_{A}$, respectively, and (12) for the determinantal representation of $\mathbf{A}^{d, W}$ give

$$
\begin{aligned}
a_{i j}^{c, \dagger, W}= & \sum_{t=1}^{m} \sum_{s=1}^{n} \frac{\sum_{\beta \in J_{r, n}\{i\}}\left|\left(\mathbf{A}^{*} \mathbf{A}\right)_{. i}\left(\mathbf{w}_{. t}^{(1)}\right)\right|_{\beta}^{\beta}}{\sum_{\beta \in J_{r, n}}\left|\mathbf{A}^{*} \mathbf{A}\right|_{\beta}^{\beta}} \\
& \times \frac{\sum_{\alpha \in I_{r_{1}, m}\{s\}}\left|(\mathbf{W} \mathbf{A})_{s .}^{k+2}\left(\bar{u}_{t .}\right)\right|_{\alpha}^{\alpha}}{\sum_{\alpha \in I_{r_{1}, n} \mid}\left|(\mathbf{W A})^{k+2}\right|_{\alpha}^{\alpha}} \frac{\sum_{\alpha \in I_{r, m}\{j j}\left|\left(\mathbf{A A}^{*}\right)_{j .}\left(\mathbf{w}_{s .}^{(2)}\right)\right|_{\alpha}^{\alpha}}{\sum_{\alpha \in I_{r, m}}\left|\mathbf{A} \mathbf{A}^{*}\right|_{\alpha}^{\alpha}},
\end{aligned}
$$

where $\mathbf{w}_{. t}^{(1)}$ is the $t$-th column of $\mathbf{W}_{1}=\mathbf{A}^{*} \mathbf{A W}, \overline{\mathbf{u}}_{t .}$ is the $t$-th row of $\overline{\mathbf{U}}=\mathbf{A}(\mathbf{W A})^{k}$, and $\mathbf{w}_{s,}^{(2)}$ is the $s$-th row of $\mathbf{W}_{2}=$ WAA $^{*}$.

Denote

$$
\phi_{i t}:=\sum_{\beta \in J_{r, n}\{i\}}\left|\left(\mathbf{A}^{*} \mathbf{A}\right)_{. i}\left(\mathbf{w}_{. t}^{(1)}\right)\right|_{\beta}^{\beta},
$$

and construct the matrix $\Phi=\left(\phi_{i t}\right)$. Then, determine

$$
\begin{aligned}
\omega_{i s} & =\sum_{t=1}^{n} \phi_{i t} \sum_{\alpha \in I_{r_{1}, n}\{s\}}\left|(\mathbf{W A})_{s .}^{k+2}\left(\bar{u}_{t .}\right)\right|_{\alpha}^{\alpha} \\
& =\sum_{\alpha \in I_{r_{1}, n}\{s\}}\left|(\mathbf{W A})_{s .}^{k+2}\left(\phi_{i .}^{(1)}\right)\right|_{\alpha}^{\alpha},
\end{aligned}
$$

where $\phi_{i .}^{(1)}$ is the $i$-th row of $\Phi_{1}=\Phi \mathbf{A}(\mathbf{W A})^{k}$ and construct the matrix $\Omega=\left(\omega_{i s}\right)$. Taking into account that $\sum_{\alpha \in I_{r, m}}\left|\mathbf{A A}^{*}\right|_{\alpha}^{\alpha}=\sum_{\beta \in I_{r, n}}\left|\mathbf{A}^{*} \mathbf{A}\right|_{\beta}^{\beta}$, and

$$
\begin{aligned}
& \sum_{s=1}^{n} \omega_{i s} \sum_{\alpha \in I_{r, m}\{j\}}\left|\left(\mathbf{A A}^{*}\right)_{j .}\left(\mathbf{w}_{s .}^{(2)}\right)\right|_{\alpha}^{\alpha} \\
& =\sum_{\alpha \in I_{r, m}\{j\}}\left|\left(\mathbf{A A}^{*}\right)_{j .}\left(\widetilde{\omega}_{i .}\right)\right|_{\alpha}^{\alpha},
\end{aligned}
$$

where $\widetilde{\omega}_{i .}$ is the $i$-th row of $\widetilde{\Omega}=\Omega \mathbf{W}_{2}=\Omega \mathbf{W A A}^{*}$, finally from (70), it follows (64).

(ii) By applying (11) for the determinantal representation of $\mathbf{A}^{d, W}$ and the same determinantal representations of $\mathbf{Q}_{A}$ and $\mathbf{P}_{A}$ as in the point (i), it is obtained that

$$
a_{i j}^{c, i, W}=\sum_{t=1}^{m} \sum_{s=1}^{n} \frac{\sum_{\beta \in J_{r, n}\{i\}}\left|\left(\mathbf{A}^{*} \mathbf{A}\right)_{. i}\left(\mathbf{w}_{. t}^{(1)}\right)\right|_{\beta}^{\beta}}{\sum_{\beta \in J_{r, n}}\left|\mathbf{A}^{*} \mathbf{A}\right|_{\beta}^{\beta}} \times \frac{\sum_{\beta \in J_{r_{1}, m i}\{t\}}\left|(\mathbf{A W})_{. t}^{k+2}\left(\overline{\mathbf{v}}_{. s}\right)\right|_{\beta}^{\beta}}{\sum_{\beta \in J_{r_{1}, m}}\left|(\mathbf{A W})^{k+2}\right|_{\beta}^{\beta}} \frac{\sum_{\alpha \in I_{r, m}\{j\}}\left|\left(\mathbf{A A}^{*}\right)_{j .}\left(\mathbf{w}_{s .}^{(2)}\right)\right|_{\alpha}^{\alpha}}{\sum_{\alpha \in I_{r, m}}\left|\mathbf{A A}^{*}\right|_{\alpha}^{\alpha}},
$$

where $\mathbf{w}_{. t}^{(1)}$ is the $t$-th column of $\mathbf{W}_{1}$ : $=\mathbf{A}^{*} \mathbf{A W}, \bar{v}_{. s}$ is the $s$-th column of $\overline{\mathbf{V}}=(\mathbf{A W})^{k} \mathbf{A}$, and $\mathbf{w}_{s \text {. }}^{(2)}$ is the $s$-th row of $\mathbf{W}_{2}:=$ WAA $^{*}$. Denote

$$
\psi_{s j}:=\sum_{\alpha \in I_{r, m}\{j\}}\left|\left(\mathbf{A A}^{*}\right)_{j .}\left(\mathbf{w}_{s .}^{(2)}\right)\right|_{\alpha}^{\alpha},
$$

and construct the matrix $\Psi=\left(\psi_{s j}\right)$. Then, introduce

$$
v_{t j}=\sum_{s=1}^{n} \sum_{\beta \in I_{r_{1}, m}\{t\}}\left|(\mathbf{A W})_{. t}^{k+2}\left(\overline{\mathbf{v}}_{. s}\right)\right|_{\beta}^{\beta} \psi_{s j}=\sum_{\beta \in I_{r_{1}, m}\{t\}}\left|(\mathbf{A W})_{. t}^{k+2}\left(\psi_{. j}^{(1)}\right)\right|_{\beta}^{\beta},
$$

where $\psi_{. j}^{(1)}$ is the $j$-th column of $\Psi_{1}=(\mathbf{A W})^{k} \mathbf{A} \Psi$, and construct the matrix $\Upsilon=\left(v_{t j}\right)$. Taking into account that

$$
\sum_{t=1}^{n} \sum_{\beta \in J_{r, n}\{i\}}\left|\left(\mathbf{A}^{*} \mathbf{A}\right)_{. i}\left(\mathbf{w}_{. t}^{(1)}\right)\right|_{\beta}^{\beta} v_{t j}=\sum_{\beta \in \epsilon_{r, n}\{i\}}\left|\left(\mathbf{A}^{*} \mathbf{A}\right)_{. i}\left(\widetilde{v}_{. j}\right)\right|_{\beta}^{\beta},
$$

where $\widetilde{v}_{. j}$ is the $j$-th column of $\widetilde{Y}=\mathbf{A}^{*} \mathbf{A W} \Upsilon$, finally from (74), it follows (67).

Theorem 7 gives determinantal representations of the WCMP inverse. For better understanding, the algorithm of its finding, for example, in Theorem 7 with the Case (i), is presented The algorithm by Theorem 7 with Case (ii) can be constructed similarly.

\section{Algorithm 2.}

(1) Compute the matrix $\mathbf{W}_{1}=\mathbf{A}^{*} \mathbf{A W}$. 
(2) Find $\phi_{i t}$ by (66) for all $i, t=1, \ldots, n$ and construct the matrix $\Phi=\left(\phi_{i t}\right)$.

(3) Compute the matrix $\Phi_{1}=\Phi \mathbf{A}(\mathbf{W A})^{k}$.

(4) By (65), find $\omega_{i s}$ for all $i=1, \ldots, m$ and $s=1, \ldots, n$, and construct the matrix $\Omega=\left(\omega_{i s}\right)$.

(5) Compute the matrix $\widetilde{\Omega}=\Omega \mathbf{W A A}$.

(6) Finally, find $a_{i j}^{c, \uparrow, W}$ by (64) for all $i=1, \ldots, m$ and $j=1, \ldots, n$.

\section{An Example}

In this section, an example is given to illustrate the results. Given the matrices

$$
\begin{aligned}
\mathbf{A} & =\left[\begin{array}{ccc}
0 & \mathbf{i} & \mathbf{i} \\
\mathbf{i} & 0 & \mathbf{i} \\
1 & -1 & 0 \\
1 & 1 & 2
\end{array}\right], \\
\mathbf{W} & =\left[\begin{array}{cccc}
\mathbf{i} & 0 & 1 & 0 \\
-1 & \mathbf{i} & 0 & 1 \\
\mathbf{i}-1 & \mathbf{i} & 1 & 1
\end{array}\right] .
\end{aligned}
$$

Since

$$
\begin{aligned}
& \mathbf{V}=\mathbf{A W}=\left[\begin{array}{cccc}
-1-2 \mathbf{i} & -2 & \mathbf{i} & 2 \mathbf{i} \\
-2-\mathbf{i} & -1 & 2 \mathbf{i} & \mathbf{i} \\
1+\mathbf{i} & -\mathbf{i} & 1 & -1 \\
-3+3 \mathbf{i} & 3 \mathbf{i} & 3 & 3
\end{array}\right], \\
& \mathbf{U}=\mathbf{W A}=\left[\begin{array}{ccc}
1 & -2 & -1 \\
0 & 1-\mathbf{i} & 1-\mathbf{i} \\
1 & -1-\mathbf{i} & -\mathbf{i}
\end{array}\right],
\end{aligned}
$$

$$
\begin{aligned}
\widetilde{\Omega} & =\mathbf{\Omega} \mathbf{W A A}^{*}=\left[\begin{array}{cccc}
0 & -54+54 \mathbf{i} & -54-54 \mathbf{i} & -54-54 \mathbf{i} \\
-54+54 \mathbf{i} & 0 & 54+54 \mathbf{i} & -54-54 \mathbf{i} \\
-54+54 \mathbf{i} & -54+54 \mathbf{i} & 0 & -108-108 \mathbf{i}
\end{array}\right], \\
\mathbf{A A}^{*} & =\left[\begin{array}{cccc}
2 & 1 & -\mathbf{i} & 3 \mathbf{i} \\
1 & 2 & \mathbf{i} & 3 \mathbf{i} \\
\mathbf{i} & -\mathbf{i} & 2 & 0 \\
-3 \mathbf{i} & 3 \mathbf{i} & 0 & 6
\end{array}\right]
\end{aligned}
$$

$\mathrm{rkV}=\mathrm{rkV}^{2}=2$, and $\mathrm{rk}^{2}=\mathrm{rk} \mathbf{U}=2$, then $k=\max$ $\{$ Ind $(\mathbf{A W})$, Ind $(\mathbf{W A})\}=1$.

The weighted DMP inverse is obtained from Algorithm 1.

(1) Computing the matrix $\widehat{\mathbf{U}}=\mathbf{U}^{2}$ and $\mathbf{A A}^{*}$,

$$
\begin{aligned}
\mathbf{U}^{2} & =\left[\begin{array}{ccc}
0 & -3+3 \mathbf{i} & -3+3 \mathbf{i} \\
1-\mathbf{i} & -2-2 \mathbf{i} & -1-3 \mathbf{i} \\
1-\mathbf{i} & -5+\mathbf{i} & -4
\end{array}\right], \\
\mathbf{U}^{3} & =\left[\begin{array}{ccc}
-3+3 \mathbf{i} & 6+6 \mathbf{i} & 3+9 \mathbf{i} \\
-4 \mathbf{i} & -8+6 \mathbf{i} & -8+2 \mathbf{i} \\
-3-\mathbf{i} & -2+12 \mathbf{i} & -5+11 \mathbf{i}
\end{array}\right] .
\end{aligned}
$$

(2) By (51), $\omega_{i s}$ for all $i, s=1, \ldots, n$ is found. So,

$$
\begin{aligned}
\omega_{i s}:= & \sum_{\alpha \in I_{2,3}\{1\}}\left|(\mathbf{W A})_{1 .}^{3}\left(\widehat{\mathbf{u}}_{1 .}\right)\right|_{\alpha}^{\alpha}=\left|\begin{array}{cc}
0 & -3+3 \mathbf{i} \\
-4 \mathbf{i} & -8+6 \mathbf{i}
\end{array}\right| \\
& +\left|\begin{array}{cc}
0 & -3+3 \mathbf{i} \\
-3-\mathbf{i} & -5+11 \mathbf{i}
\end{array}\right|=-24-6 i .
\end{aligned}
$$

Continuing similarly, it is obtained that

$$
\mathbf{\Omega}=\left[\begin{array}{ccc}
-24-6 \mathbf{i} & 12-24 \mathbf{i} & -12-30 \mathbf{i} \\
12+12 \mathbf{i} & -6-6 \mathbf{i} & 6+6 \mathbf{i} \\
-12+6 \mathbf{i} & 6-30 \mathbf{i} & -6-24 \mathbf{i}
\end{array}\right]
$$

(3) Compute the matrix 
and the values

$$
\sum_{\beta \in J_{2,4}}\left|\mathbf{A A}^{*}\right|_{\beta}^{\beta}=27, \sum_{\alpha \in I_{, n}}\left|(\mathbf{W A})^{3}\right|_{\alpha}^{\alpha}=-54-54 \mathbf{i} .
$$

(4) Finally, $a_{i j}^{d, \dagger, W}$ by (50) for all $i=1, \ldots, 4$ and $j=$ $1,2,3$ is found. So,

$$
\begin{aligned}
a_{11}^{d, \dagger, W}= & \frac{\sum_{\alpha \in I_{2,4}\{1\}} r \operatorname{det}_{1}\left(\left(\mathbf{A A}^{*}\right)_{1 .}\left(\widetilde{\omega}_{1 .}\right)\right)_{\alpha}^{\alpha}}{\left.\sum_{\beta \in I_{2,4}}\left|\mathbf{A A}^{*}\right|_{\alpha}^{\alpha} \sum_{\alpha \in I_{2,3}} \mid \mathbf{W A}\right)\left.^{3}\right|_{\alpha} ^{\alpha}} \\
= & \frac{1}{27(-54-54 \mathbf{i})}\left(\left|\begin{array}{cc}
0 & -54+54 \mathbf{i} \\
1 & 2
\end{array}\right|+\left|\begin{array}{cc}
0 & -54+54 \mathbf{i} \\
i & 2
\end{array}\right|\right. \\
& \left.+\left|\begin{array}{cc}
0 & -54-54 \mathbf{i} \\
-3 \mathbf{i} & 6
\end{array}\right|\right)=\frac{\mathbf{i}}{9} .
\end{aligned}
$$

Continuing similarly, it is obtained that

$$
\mathbf{A}^{d, \dagger, W}=\frac{1}{9}\left[\begin{array}{cccc}
\mathbf{i} & -2 \mathbf{i} & 3 & 1 \\
-2 \mathbf{i} & \mathbf{i} & -3 & 1 \\
-\mathbf{i} & -\mathbf{i} & 0 & 2
\end{array}\right] .
$$

It is easy to verify that $\mathbf{X}=\mathbf{A}^{d, \dagger, W}$ from (86) with the given matrices (86) is the solution to equation (45).

Similarly, the WMPD inverse can be found:

$$
\mathbf{A}^{\dagger, d, W}=\frac{1}{6}\left[\begin{array}{cccc}
-3+\mathbf{i} & -3+3 \mathbf{i} & 4 & 3+3 \mathbf{i} \\
-2 \mathbf{i} & 0 & -2 & 0 \\
-3-\mathbf{i} & -3+3 \mathbf{i} & 2 & 3+3 \mathbf{i}
\end{array}\right] .
$$

\section{Conclusions}

In this paper, new notions of the weighted core-EP left inverse and the weighted MPD inverse that are dual to the weighted core-EP (right) inverse and the weighted DMP inverse, respectively, are introduced and explored. Using their determinantal representations, the direct methods of computing the weighted right and left core-EP, DMP, MPD, and CMP inverses are given.

\section{Data Availability}

No data were used to support this study.

\section{Conflicts of Interest}

The author declares that there are no conflicts of interest.

\section{References}

[1] R. E. Cline and T. N. E. Greville, "A Drazin inverse for rectangular matrices," Linear Algebra and Its Applications, vol. 29, pp. 53-62, 1980.
[2] A. Hernández, M. Lattanzi, and N. Thome, "On some new pre-orders defined by weighted Drazin inverses," Applied Mathematics and Computation, vol. 282, pp. 108-116, 2016.

[3] A. Herrero, F. J. Ramírez, and N. Thome, "Relationships between different sets involving group and Drazin projectors and nonnegativity," Linear Algebra and Its Applications, vol. 438, no. 4, pp. 1688-1699, 2013.

[4] P. S. Stanimirović, V. N. Katsikis, and H. Ma, "Representations and properties of the $\mathrm{W}$-weighted Drazin inverse," Linear and Multilinear Algebra, vol. 65, no. 6, pp. 1080-1096, 2017.

[5] N. Thome and Y. Wei, "Generalized inverses and a block-rank equation," Applied Mathematics and Computation, vol. 141, no. 2-3, pp. 471-476, 2003.

[6] Y. Wei, "Integral representation of the W-weighted Drazin inverse," Applied Mathematics and Computation, vol. 144, no. 1, pp. 3-10, 2003.

[7] Y. Wei, "A characterization for the W-weighted Drazin inverse and a Cramer rule for the $\mathrm{W}$-weighted Drazin inverse solution," Applied Mathematics and Computation, vol. 125, no. 2-3, pp. 303-310, 2002.

[8] Z. Al-Zhour, A. Kiliçman, and M. H. Abu-Hassa, "New representations for weighted Drazin inverse of matrices," International Journal of Mathematical Analysis, vol. 1, no. 15, pp. 697-708, 2007.

[9] R. Penrose, "A generalized inverse for matrices," Mathematical Proceedings of the Cambridge Philosophical Society, vol. 51, no. 3, pp. 406-413, 1955.

[10] G. Wang, Y. Wei, and S. Qiao, Generalized Inverses: Theory and Computations, Springer, Berlin, Germany, 2nd edition, 2018.

[11] Y. Wei, P. S. Stanimirović, and M. D. Petković, Numerical and Symbolic Computations of Generalized Inverses, World Scientific Publishing Co. Pte. Ltd., Singapore, 2018.

[12] O. M. Baksalary and G. Trenkler, "Core inverse of matrices," Linear and Multilinear Algebra, vol. 58, no. 6, pp. 681-697, 2010.

[13] S. B. Malik and N. Thome, "On a new generalized inverse for matrices of an arbitrary index," Applied Mathematics and Computation, vol. 226, pp. 575-580, 2014.

[14] S. Xu, J. Chen, and X. Zhang, "New characterizations for core inverses in rings with involution," Frontiers of Mathematics in China, vol. 12, no. 1, pp. 231-246, 2017.

[15] K. M. Prasad and K. S. Mohana, "Core-EP inverse," Linear and Multilinear Algebra, vol. 62, no. 6, pp. 792-802, 2014.

[16] R. B. Bapat, K. P. S. Bhaskara Rao, and K. M. Prasad, "Generalized inverses over integral domains," Linear Algebra and Its Applications, vol. 140, pp. 181-196, 1990.

[17] K. P. S. Bhaskara Rao, "On generalized inverses of matrices over integral domains," Linear Algebra and Its Applications, vol. 49, pp. 179-189, 1983.

[18] O. M. Baksalary and G. Trenkler, "On a generalized core inverse," Applied Mathematics and Computation, vol. 236, pp. 450-457, 2014.

[19] M. Mehdipour and A. Salemi, "On a new generalized inverse of matrices," Linear and Multilinear Algebra, vol. 66, no. 5, pp. 1046-1053, 2018.

[20] J. Chen, H. Zhu, P. Patricio, and Y. Zhang, "Characterizations and representations of core and dual core inverses," Canadian Mathematical Bulletin, vol. 60, no. 2, pp. 269-282, 2017.

[21] D. E. Ferreyra, F. E. Levis, and N. Thome, "Maximal classes of matrices determining generalized inverses," Applied Mathematics and Computation, vol. 333, pp. 42-52, 2018. 
[22] Y. Gao and J. Chen, "Pseudo core inverses in rings with involution," Communications in Algebra, vol. 46, no. 1, pp. 38-50, 2018.

[23] X. Liu and N. Cai, "High-order iterative methods for the DMP inverse," Journal of Mathematics, vol. 2018, Article ID 8175935, 6 pages, 2018.

[24] H. Ma and P. S. Stanimirović, "Characterizations, approximation and perturbations of the core-EP inverse," Applied Mathematics and Computation, vol. 359, pp. 404-417, 2019.

[25] H. Ma and T. Li, "Characterizations and representations of the core inverse and its applications," Linear and Multilinear Algebra, pp. 1-11, 2019.

[26] H. Ma, X. Gao, and P. S. Stanimirović, "Characterizations, iterative method, sign pattern and perturbation analysis for the DMP inverse with its applications," Applied Mathematics and Computation, vol. 378, Article ID 125196, 2020.

[27] K. M. Prasad and M. D. Raj, "Bordering method to compute core-EP inverse," Special Matrices, vol. 6, no. 1, pp. 193-200, 2018.

[28] D. S. Rakić, N. Č. Dinčić, and D. S. Djordjević, "Group, Moore-Penrose, core and dual core inverse in rings with involution," Linear Algebra and Its Applications, vol. 463, pp. 115-133, 2014.

[29] H. Wang, "Core-EP decomposition and its applications," Linear Algebra and Its Applications, vol. 508, pp. 289-300, 2016.

[30] M. Zhou, J. Chen, T. Li, and D. Wang, "Three limit representations of the core-EP inverse," Filomat, vol. 32, no. 17, pp. 5887-5894, 2018.

[31] I. I. Kyrchei, "Determinantal representations of the core inverse and its generalizations with applications," Journal of Mathematics, vol. 2018, Article ID 8175935, 6 pages, 2018.

[32] I. Kyrchei, "Determinantal representations of the quaternion core inverse and its generalizations," Advances in Applied Clifford Algebra, vol. 29, no. 5, p. 104, 2019.

[33] I. I. Kyrchei, "Cramer's rule for quaternionic systems of linear equations," Journal of Mathematical Sciences, vol. 155, no. 6, pp. 839-858, 2008.

[34] I. I. Kyrchei, "The theory of the column and row determinants in a quaternion linear algebra," in Advances in Mathematics Research, A. R. Baswell, Ed., vol. 15, pp. 301-359, Nova Science Publishers, New York, NY, USA, 2012.

[35] D. E. Ferreyra, F. E. Levis, and N. Thome, "Revisiting the core EP inverse and its extension to rectangular matrices," Quaestiones Mathematicae, vol. 41, no. 2, pp. 265-281, 2018.

[36] Y. Gao, J. Chen, and P. Patrício, "Representations and properties of the $\mathrm{W}$-weighted core-EP inverse," Linear and Multilinear Algebra, pp. 1-15, 2018.

[37] H. Ma, "A characterization and perturbation bounds for the weighted core-EP inverse," Quaestiones Mathematicae, pp. 1-11, 2019.

[38] D. Mosić, C. Deng, and H. Ma, "On a weighted core inverse in a ring with involution," Communications in Algebra, vol. 46, no. 6, pp. 2332-2345, 2018.

[39] D. Mosić, "Weighted core-EP inverse of an operator between Hilbert spaces," Linear and Multilinear Algebra, vol. 67, no. 2, pp. 278-298, 2019.

[40] L. Meng, "The DMP inverse for rectangular matrices," Filomat, vol. 31, no. 19, pp. 6015-6019, 2017.

[41] D. Mosić, "The CMP inverse for rectangular matrices," Aequationes Mathematicae, vol. 92, no. 4, pp. 649-659, 2018.

[42] P. S. Stanimirovic, "General determinantal representation of pseudoinverses of matrices," Matematički Vesnik, vol. 48, pp. 1-9, 1996.
[43] Y. Yu and Y. Wei, "Determinantal representation of the generalized inverse $\backslash$ bi $A \_\{T, S\}\{(2)\}$ over integral domains and its applications," Linear and Multilinear Algebra, vol. 57, no. 6, pp. 547-559, 2009.

[44] I. I. Kyrchei, "Analogs of the adjoint matrix for generalized inverses and corresponding Cramer rules," Linear and Multilinear Algebra, vol. 56, no. 4, pp. 453-469, 2008.

[45] I. I. Kyrchei, "Determinantal representations of the Moore-Penrose inverse over the quaternion skew field and corresponding Cramer's rules," Linear and Multilinear Algebra, vol. 59, no. 4, pp. 413-431, 2011.

[46] I. Kyrchei, "Explicit formulas for determinantal representations of the Drazin inverse solutions of some matrix and differential matrix equations," Applied Mathematics and Computation, vol. 219, no. 14, pp. 7632-7644, 2013.

[47] I. Kyrchei, "Determinantal representations of the Drazin inverse over the quaternion skew field with applications to some matrix equations," Applied Mathematics and Computation, vol. 238, pp. 193-207, 2014.

[48] I. I. Kyrchei, "Cramer's rule for generalized inverse solutions," in Advances in Linear Algebra Research, I. I. Kyrchei, Ed., pp. 79-132, Nova Science Publishers, New York, NY, USA, 2015.

[49] I. Kyrchei, "Determinantal representations of the W-weighted Drazin inverse over the quaternion skew field," Applied Mathematics and Computation, vol. 264, pp. 453-465, 2015.

[50] I. I. Kyrchei, "Explicit determinantal representation formulas of W-weighted Drazin inverse solutions of some matrix equations over the quaternion skew field," Mathematical Problems in Engineering, vol. 2016, Article ID 8673809, 13 pages, 2016. 